\title{
Respirators evaluated by fit testing
}

\section{To the Editor:}

The COVID-19 pandemic has led to challenges in the steady supply of single-use masks (respirators) [1] and many hospitals are faced with using new types of respirators to avoid stock-outs. While certified FFP2/KN95 respirators have the ability to prevent airborne infections, like COVID-19, influenza or tuberculosis, the performance of the respirators depends on tight sealing of nose and mouth.

We performed seal checks with five types of respirators designated to have filtering abilities compliant with the FFP2 standard (Polmaska Donald and X200 (Oxyline, Pabianice, Poland), Aura 1862+ (3M, Neuss, Germany), and AP-81001 and AP-83001 (MAXK Zhejiang Aopeng Industry and Trading Co. Ltd, Zhejiang, China)) and four different KN95 respirators with ear straps prospectively on healthcare workers in a 83-bed hospital setting by fit testing (FT 30; 3M). The fit test assesses how well a respirator seals off the face via taste perception of a sodium saccharine aerosol by the test person, where a perceived taste indicates a leak in the seal around mouth and nose [2].

Only the "Polmaska Donald" passed the fit test perfectly (no taste perceived in 40 out of 40 workers, 100\%); the Aura 1862+, X200, AP-81001 and AP-83001 passed fit testing in 80 out of 97 (82.5\%), 48 out of $66(72.7 \%), 31$ out of $46(67.4 \%)$ and seven out of $31(22.6 \%)$ healthcare workers. KN95 masks failed fit testing in all (82 out of $82,100 \%$ ) healthcare workers evaluated (figure 1).

Respirators can vary greatly in their ability to protect the wearer by their filtering medium, and sealing of the mouth and nose tightly. Variations in human facial anatomy must be accounted for by consequently fit testing respirators for all healthcare workers in facilities at risk for airborne transmitted infections like COVID-19, influenza or tuberculosis.

@ERSpublications

FFP2/KN95 respirators can vary greatly in their ability to protect the wearer. Fit testing of respirators should be performed by all healthcare workers at risk for airborne transmitted diseases like \#COVID19, \#influenza or \#tuberculosis https://bit.ly/3lFuwy5

Cite this article as: Kleinjohann L, Lange C. Respirators evaluated by fit testing. ERJ Open Res 2020; 6: 00581-2020 [https://doi.org/10.1183/23120541.00581-2020].

\section{Lukas Kleinjohann $^{1,2}$ and Christoph Lange ${ }^{1,3,4}$}

${ }^{1}$ Clinical Infectious Diseases, Research Center Borstel, Borstel, Germany. ${ }^{2}$ University Medical Center Hamburg-Eppendorf, Hamburg, Germany. ${ }^{3}$ International Health/Infectious Diseases University of Lübeck, Lübeck, Germany. ${ }^{4}$ German Center for Infection Research (DZIF) Clinical Tuberculosis Unit, Borstel, Germany.

Correspondence: Christoph Lange, Clinical Infectious Diseases, Research Center Borstel, Parkallee 35, 23845 Borstel, Germany. E-mail: clange@fz-borstel.de

Received: 14 Aug 2020 | Accepted: 14 Aug 2020

Support statement: C. Lange is supported by the German Center for Infection Research (DZIF). Funding information for this article has been deposited with the Crossref Funder Registry.

Conflict of interest: L. Kleinjohann has nothing to disclose. C. Lange reports personal fees from Chiesi, Gilead, Janssen, Novartis, Oxfordimmunotec and Insmed, outside the submitted work. 

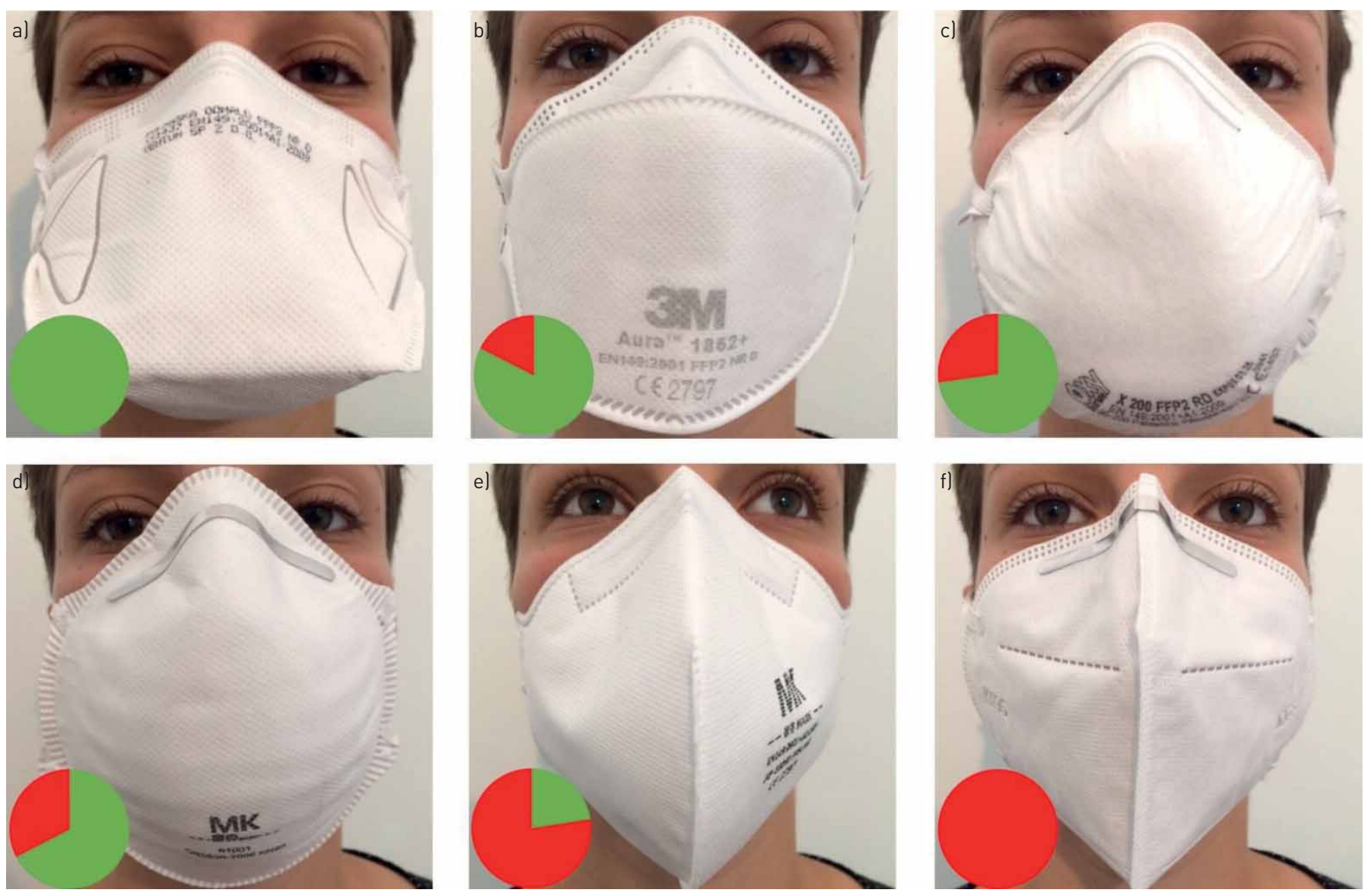

FIGURE 1 Different respirators evaluated by fit testing for tight sealing of nose and mouth. a) Polmaska Donald; b) Aura 1862+; c) X200; d) AP-81001; e) AP-83001; f) KN95 type. Pie charts show the percentages of tight sealing (green) and nonsealing (red) respirators in healthcare workers.

\section{References}

1 Tabah A, Ramanan M, Laupland KB, et al. Personal protective equipment and intensive care unit healthcare worker safety in the COVID-19 era (PPE-SAFE): An international survey. J Crit Care 2020; 59: 70-75.

2 United States Department of Labor. 1910.134 App A - Fit Testing Procedures (Mandatory). www.osha.gov/lawsregs/regulations/standardnumber/1910/1910.134AppA. Date last assessed: 8 July 2020. 